\title{
Visualization of synaptic vesicle dynamics with fluorescence proteins
}

\author{
Wang Li, Chunyang Geng, Bo Liu \\ Department of Biomedical Engineering, Dalian University of Technology, Dalian (Liaoning), China
}

\begin{abstract}
Synaptic vesicle (SV) will be transported to the bouton along the axon, once it is formed in a cell body. After docking in the active zone, neurotransmitters will be released upon the stimulation, and then transmission of chemical signals will be initiated. Presently, many advanced technologies and burgeoning molecular sensors are being used to explore the synaptic transmission. These studies provide a new sight into the presynaptic structure and its function. The present review summarizes the application of fluorescent proteins (FPS) for SV tracking and recycling. Some FPS and relevant imaging technologies such as fluorescence resonance energy transfer (FRET), fluorescence recovery after photobleaching (FRAP) and fluorescence lifetime imaging microscopy (FLIM), are introduced here. In addition, some examples are also analyzed to visualize the dynamics of SVs in living cells with the help of some FPS.
\end{abstract}

Key words: synaptic vesicle, fluorescent proteins, fluorescent technologies, dynamics.

\section{Introduction}

Synaptic vesicles (SVS) play essential roles in information transferring such as visual sense, auditory sense and even emotions. The small spherical SV fuses with presynaptic membrane and releases its contents of the neurotransmitter (exocytosis). Subsequently, the neurotransmitters diffuse across the cleft between pre- and postsynaptic membranes, leading to the activation or inhibition of the postsynaptic neuron. Consequently, an intact information transfer is accomplished during this period. At the same time, the components of SVs are recovered to the presynaptic membrane for next transfer preparation (endocytosis).

Researchers began their studies on SVs by electrophysiological ways because some electrophysio- logical parameters such as current across the membrane would be changed during exocytosis or endocytosis [97]. Therefore, ion fluxes through neurotransmitter-gated channels can be measured electrically via recording the whole cell from soma or dendrites of postsynaptic neurons $[7,20]$. Similarly, SV recycling [98], neurotransmitters secretion [40], and membrane fusion [65], have also been detected via electric current, capacitance and some other electrophysiological parameters. However, these methods usually face some difficulties in reporting the SVs release from an individual release site or synapse, and they cannot show the complete spatial information about the origin and destination [42].

As the fluorescent technology develops, biological experts can employ the fluorescent probes to 
report the neurotransmitters release at the level of individual synapse, and collect more information about vesicle tracking and fusion [49]. One of these probes adopted fluid phase markers such as fluorescent dyes to visualize membrane recycling through two basic formats (i.e., dye uptake and dye release) $[3,41]$. Dye-based methods mainly focus on lipid cycling in an active-dependent manner. Thanks to rapid uptake and release of the dye, the same vesicle was found to be reused several times during routine neurotransmission with the help of FM1-43 [4]. These fluorescent dyes have high molar absorptivity, strong fluorescence and great photostability [23]. Interestingly, they also become brighter distinctly once their tail domains partition into the membrane [37]. For example, FM1-43 increases fluorescence 100 times brighter in the membrane than in the aqueous solution [51]. Such changes in fluorescence intensity provide possibility for analyzing SVs fusion and internalization. Although it is easy to mark SVs with dyes [26], these measurements are still limited by the non-specificity of dyes since they stain the membrane randomly [86]. Furthermore, SVs release neurotransmitters in a millisecond time scale, which makes it tough for fluorescent dyes to be detected as their fluorescence changes in seconds [42]. The worst thing is the toxicity of dyes. For example, FM1-43 behaves as a permeant blocker of mechanosensitive channels in sensory neurons [21,27].

Contrarily, some other probes take advantage of genetic expression of SV protein tagged with fluorescent proteins (FPs) to measure the kinetics of vesicles intuitively [52]. These probes focus on proteins cycling without any perturbation to the original function mostly, since they are constructed on DNA by subcloning and expressed by cells themselves after transfection. Therefore, SV-related proteins could be labeled specifically and detected in real time with the help of FPs. The kinetics of SVs with an emphasis on the application of fluorescent proteins to SVs is introduced below.

\section{Fluorescent proteins and relevant imaging technologies}

FPs technology has been confirmed to be a very useful tool for imaging living cells since its vast application in the research field in recent years. The first FP found in jellyfish Aequorea victoria is green fluorescence protein (GFP) with two different chromo- phores [12,74]. A mass of FPs have been developed later, including short wavelength FPs derived from ECFP and long wavelength FPs derived from DsRed [92]. They supply various colored tools on a molecular level for the visualization of single or multiple target molecules in living cells [85]. These FPs can also be used to track the movement of vesicle-associated proteins in real time. For example, it is easy to view the distribution of synapsin la during stimulation by using EGFP-synapsin la fusion protein generated through subcloning synapsin la into pEGFP-C1 vector [18]. Some variants of FPs are found sensitive to regional changes like $\mathrm{pH}$, ionic concentration or oxidability [92]. For example, pHluorin is a widely used $\mathrm{pH}$-dependent variant of GFP whose fluorescence is quenched at acidic $\mathrm{pH}$ while recovered after being exposed to near-neutral $\mathrm{pH}$ in the solution [2]. As the $\mathrm{pH}$ of SV lumen changes from acidic to near-neutral during exocytosis and re-acidic after endocytosis, the changes in fluorescence intensity caused by varying $\mathrm{pH}$ can reflect SVs' recycling and can be used for quantitative assessment [50,93]. Moreover, a set of similar FPs have been attained via different directed point mutations $[10,61,89]$. Such properties have provided us with efficient tools for detecting ionic fluxes or $\mathrm{pH}$ changes in vivo.

With the development of diversified FPs, advanced imaging technologies based on FPs have been put forward, including fluorescence resonance energy transfer (FRET), fluorescence recovery after photobleaching (FRAP) and fluorescence lifetime imaging microscopy (FLIM). FRET is a quantum mechanical phenomenon where a donor FP transfers energy to an acceptor FP within a close distance if donor's emission spectrum overlaps the excitation spectrum of the acceptor [53]. FRET efficiency, which is calculated by the acceptor/ donor emission ratio, has a pally relationship with the distance and relative angles between the donor and the acceptor. Thus, the conformational changes of a target molecule, which has been inserted into FRET pair proteins, result in a FRET signal which can be utilized to explore the interaction between two presynaptic proteins [16]. For instance, with EGFP and tdTomato fusing to presynaptic protein dNSF1 and synaptosomal-associated protein of $25 \mathrm{kDa}$ (SNAP-25) respectively, FRET efficiency increased once the two target proteins interacted with each other and shortened their distances [99]. FRAP is a process where the fluorescence signals are selectively photobleached and recovered in the same region over a period of 
time. Usually, this technology is usually used to analyze the mobility of fluorescent molecules [9]. For example, the mobility of dNSF1 tagged with EGFP could be estimated through the speed of fluorescence recovery after photobleaching [99]. FLIM is a technique for visualizing the lifetime of the excitation state of the spatially distributed fluorescence molecules [91]. This technology can be applied to determine the interaction between the donor and the acceptor along with FRET because the fluorescence lifetime of donor changes during the occurrence of FRET $[90,92]$.

\section{Vesicle associated protein: fluorescent tags}

SVs are released at the presynaptic active zone mainly in three steps: docking, priming and release [79]. The precursor vesicle is formed in the neural cell body and internalizes SV proteins which are synthesized in endoplasmic reticulum (ER) or Golgi apparatus. Then the vesicle is transported to the synapse along the axon with a directed motion. After being placed SV proteins in the right position and embellished with cofactors or other necessary proteins, basically the first SV comes into being [70]. Cur- rently, the SV has no function until it is acidized by a proton pump called vATPase and filled with neurotransmitters [22,73]. Then SV is driven to the active zone with an unclear docking mechanism. One mode holds a view that soluble $N$-ethylmaleimide-sensitive factor attachment protein receptors (SNAREs) [19], which are the core of the docking-fusion complex comprised of syntaxin [96], synaptobrevin (also called vesicle-associated membrane protein, VAMP) [82], and SNAP-25 [28], tether vesicles to the presynaptic plasma membrane via interactions of proteins (Fig. 1) $[58,69,75]$. In addition, Rab3-interacting molecule (RIM) helps the vesicle to connect with $\mathrm{Ca}^{2+}$ channel protein [39]. Complexin [59], which stabilizes SNARES complex and RIM, promotes the process of dock and prepares the vesicle for release [80]. Under proper conditions like the sharp entry of calcium, SVs fusion and release may take place with the help of synaptotagmin [15,81].

Many other proteins have also taken part in the exocytosis, for example, RIM-binding protein (RIM-BP) [54], mammalian homologue of Caenorhabditis elegans unc-13-1 (Munc13-1) [36], liprin [6] and so on.

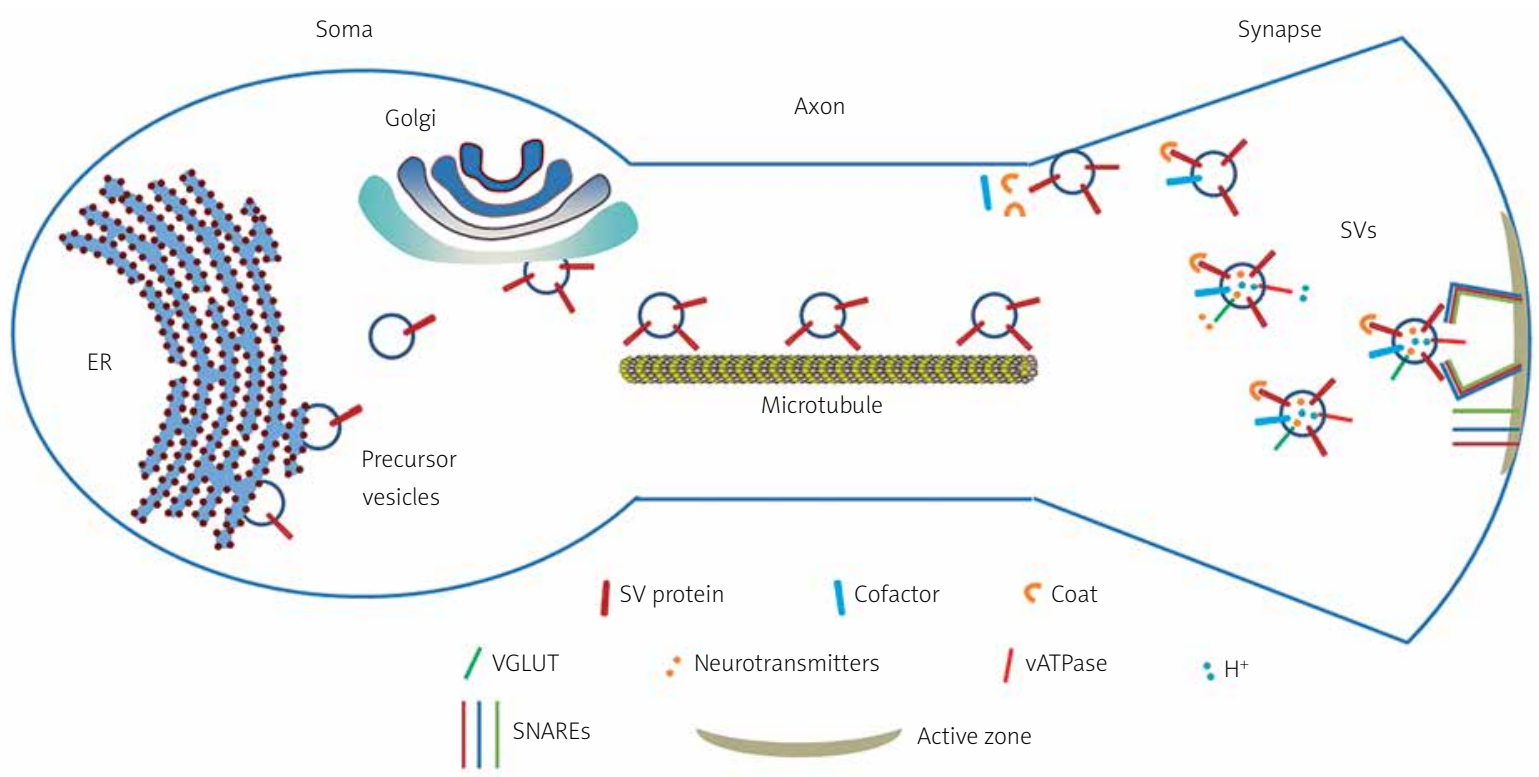

Fig. 1. SVs are transported from soma to synapse $[19,22,70,73]$. SVs are first formed in neural cell body (termed precursor vesicle), and modified with SV proteins in ER and Golgi. Then they are transported along with microtubule toward presynaptic terminal. Within synapse, SVs are formed basically after sorting of SV components and being embellished with necessary proteins like cofactors and coats. After that, SVs are acidized and filled with neurotransmitters before being tethered to active zone for release, with the help of SNAREs, and maybe some other proteins like RIM and Munc13-1. 
These proteins play important roles in regulating SVs' cluster, transport and recycle. In this section, some FPs-dependent sensors and FPs-associated technologies will be introduced along with some main presynaptic proteins in order to give an intuitionistic description of the kinetics of SVs.

\section{Synaptobrevin/VAMP}

VAMP is identified as an important regulator of vesicular transport with seven kinds of isoforms, VAMP-1/2/3/4/5/7/8, which are expressed differently in the central nervous system [84]. Previous studies have reported that VAMP was involved in rapid and slow endocytosis together with SNAP-25 and syntaxin [96]. Additionally, it may migrate with SVs synchronously during SVs' activity because of its location on the SV membrane [38]. Thanks to this property, VAMP is usually used to explore the mobil- ity of SVs. For example, synaptobrevin 2 tagged with EGFP (syb2-EGFP) was applied to measure the speed of moving SVs cluster by taking images of syb2-EGFP labeled material moving in intersynaptic areas [77]. Furthermore, by using DsRed2-synaptobrevin2, the formation of presynaptic vesicle clusters could be seen during synapse maturation, and its regulation by $\mathrm{N}$-cadherin could be visualized with EGFP-VAMP2 through the technology of FRAP [78]. Recently, VAMP-pHluorin (also named as synaptobrevin-pHluorin, $\mathrm{spH}$ ) was also used to study the mobility of recycling SVs in living hippocampal nerve terminal combined with Atto647N-conjugated nanobodies [19]. Some researchers even took advantage of VAMP-pHluorin to test the effects of different genes on SV cycling [30]. VAMP-pHluorin is a common fluorescent probe to monitor the exo- and/or endocytosis [13]. Its pH-sensitive pHluorin lies in the SV lumen

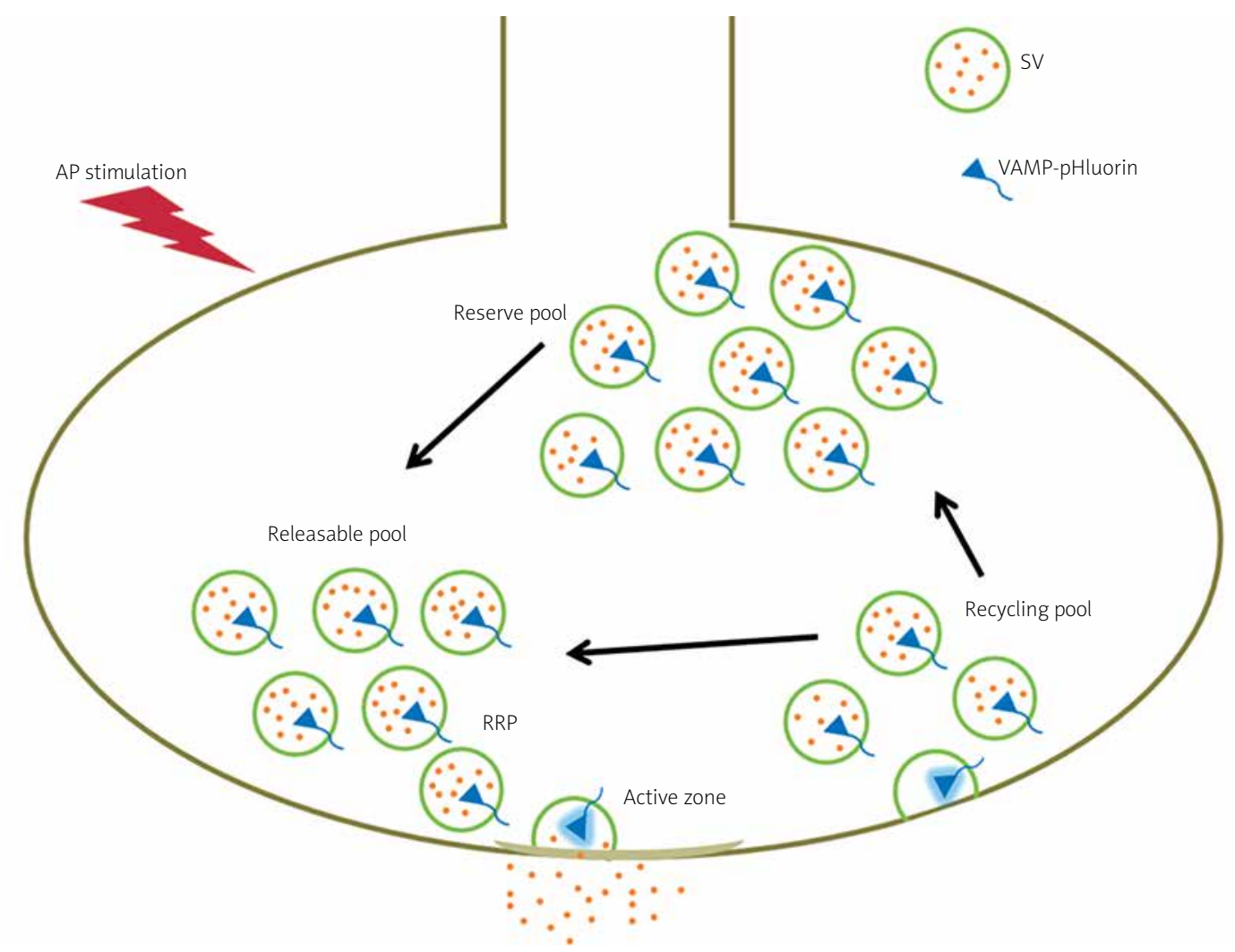

Fig. 2. Balance between exocytosis and endocytosis revealed by VAMP-pHluorin [2,24]. VAMP-pHluorin was used to monitor the accumulation of SVs on the presynaptic membrane and its fluorescence increased about 20 times when exocytosis happened. According to the changes in fluorescence intensity induced by different frequencies of stimulation at physiological temperature, it was concluded that the releasable SV pool was not depleted significantly under stimulation up to $10 \mathrm{~Hz}$. This result indicated that the other two pools supplemented releasable pool with SVs to maintain the balance between exocytosis and endocytosis. 
which has an acidic environment, so it has no fluorescence until it is exposed to the near-neutral $\mathrm{pH}$ environment after vesicles fuse to membrane. Therefore, a change in fluorescence intensity can assess the synaptic transmission. Surprisingly, fluorescence intensity changed inhomogenously throughout an individual presynaptic terminal no matter which frequency of stimulation was applied. Further experiment revealed that changes in fluorescence intensity had a significant linear correlation with the abundance of local SVs [94]. According to the three-pool model, local vesicles come from readily releasable pool (RRP), recycling pool and reserve pool. RRP has the highest probability for releasing vesicles at each bouton while the other two pools act as replenishments [2]. With the help of $\mathrm{spH}$, it has been found that the releasable SV pool was not depleted significantly under action potential (AP) stimulation (less than $10 \mathrm{~Hz}$ ) at physiological temperature, indicating a balance between the exocytosis and endocytosis (Fig. 2) [24]. It was reported that VAMP also played its role in SVs fusion. Xia et al. developed a FRETbased biosensor comprised of YFP-synaptobrevin and CFP-SNAP-25. They found that the FRET efficiency increased with given stimulation, suggesting the assembly of SNAREs by synaptobrevin and SNAP-25 [95]. This assembly might help SVs get closer to the presynaptic membrane and be ready for release.

\section{Synaptophysin}

Synaptophysin is the most abundant intrinsic membrane glycoprotein of SVs with four transmembrane domains and two loops on the lumen side $[5,87]$. It has a $\mathrm{Ca}^{2+}$-binding site on the cytoplasmic side and may be involved in the $\mathrm{Ca}^{2+}$-trigger mechanism that initiates the opening of a fusion pore [17]. Currently, synaptophysin is an important marker of SV which provides reliable data for the synaptic structure [43]. Moreover, it can be used to track the movement or recycling of SVs either through FRAP with synaptophysin I-EGFP or by fusing with FPs alone (Fig. 3) $[29,63,102]$. Amazingly, it was found that two distinct vesicles were endocytosed while only one vesicle fused with the presynaptic membrane when imaging the synaptophysin-pHluorin (SypHluorin) in an individual vesicle under low-frequency stimulation [101]. This phenomenon seemed to disrupt the balance between exocytosis and endocytosis, shown by the application of spH to the vesicle pool [24]. In general perception, there are two pathways of SV recovery: one fast pathway in which the vesicles remain or are recycled around the active zone, and the other slow one, a clathrin-involved pathway [32,71]. By using SypHluorin, it was found that the slower endocytosis was accelerated and became the predominant pathway with increased stimulation frequency [101]. As a result, a new match was rebuilt between endocytosed vesicles and exocytosed vesicles.

Besides, SypHluorin was also used to measure the release possibility of SVs which was an important component of synaptic strength [57]. Other functional properties like evoked release and total vesicle pool size, were also studied upon SypHluorin applications [67]. Recently, SypHluorin has been widely used to test drugs and explore proteins function within synapses [31]. It was found that $N$-cadherin and Neuroligin 1 could significantly promote exocytosis [88], and presenilin 1 could facilitate exocytosis directly by interacting with synaptotagmin 1 [102]. In addition, synaptophysin marked with EYFP and

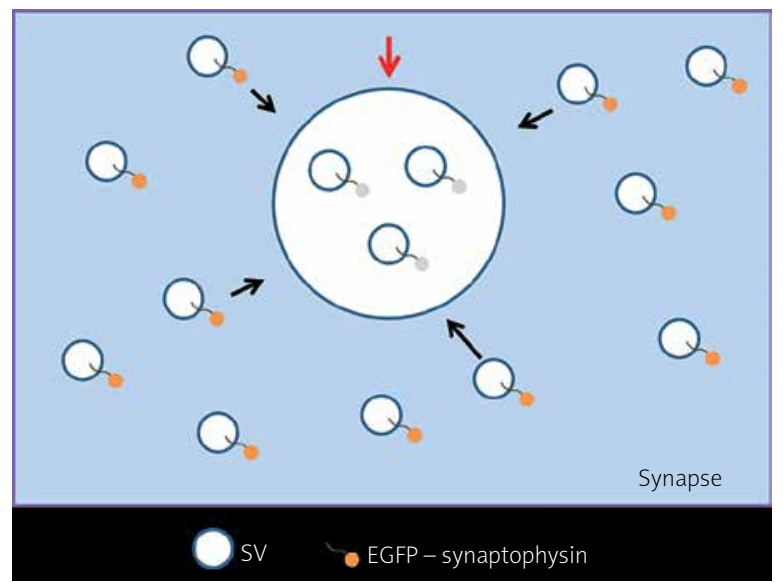

Fig. 3. Tracked SVs with EGFP-synaptophysin via the technology of FRAP $[29,63,102]$. The fluorescence of selected region of interest (ROI, the red arrow) decreased drastically during photobleaching experiments, and recovered over a period of time after bleaching because surrounding SVs marked with EGFP-synaptophysin migrated to ROI again. By recording the recovery time, the speed of SVs' movement could be measured easily. Besides, this biosensor could be used to explore the influences of proteins or protein interactions on the tracking of SVs through disturbing the expression of relevant proteins. 
ECFP-VAMP2 were designed for a FRET-based biosensor, which could detect the interaction between synaptophysin and VAMP2 during exocytosis by analyzing the changes in FRET signals [68]. Moreover, with the development of calcium indicator GCaMP [60], more methods of combining two FPs with non-overlapping color spectra, such as SyGCaMP2 and SypHluorin, have been used to monitor simultaneously the spatial and temporal relationships of calcium influx and the dynamics of SVs $[48,100]$, which makes it possible for the detection of signals in parallel.

\section{Vesicular glutamate transporter (VGLUT)}

VGLUT has three isoforms named VGLUT1/2/3 which are distributed in different places [34,77]. It participates in the probability of SVs release and synaptic plasticity because it acts as a carrier that loads the neurotransmitter into SVs [72]. The relationship between changes in calcium ion concentration and vesicle recycling has been analyzed by means of VGLUT1-mOrange2 and SyGCaMP3 [47]. It has also been discovered that $\mathrm{Ca}^{2+}$ increases the SV fusion probability but decreases the rate of SV retrieval with VGLUT-pHluorin by contrast [45]. Focusing on the single SV, Balaji and Ryan gave more details about the retrieval time. They found that the recovery occurred over a wide range of time with an average constant of 14 seconds, suggesting a stochastic process of retrieval [8]. In addition, by utilizing VGLUT-pHluorin, the increase in fluorescence was viewed after using tetrodotoxin [46], a voltage-gated $\mathrm{Na}^{+}$channel blocker. It was concluded that SVs should be released spontaneously except for responding to the action potential. Besides, the fluorescence delay was very short (less than $370 \mathrm{~ms}$ ), indicating that SVs were recycled at an extremely fast speed [46]. It was supposed that vesicles were endocytosed into the recycling pool in a faster way that SNARE-binding protein Tomosyn was involved in the regulation of dynamic partitions of SVs pool, and knockdown of Tomo1 might lead SVs to total recycling pool, including RRP and recycling pool [14].

\section{Other proteins}

FPs have also been used to explore the function of some other proteins in synaptic vesicle dynamics. Synapsin involves in managing the presynaptic reverse pool and plays an important role in synaptic short-term plasticity [62]. By using synapsin la-EYFP or synapsin Ila-EGFP with the application of FRAP, the extent of fluorescence recovery was found to be less than that in synapsin-triple-knockout neurons. It indicated that synapsins could immobilize SVs and control the mobility of the resting pool vesicle [63]. By employing the same probe, changes in fluorescence puncta could be seen clearly in the stimulated neuron. Thus it was concluded that exocytosis and endocytosis were necessary for synapsin to redistribute into the axon by analysis of fluorescence images [64]. Combined EGFP-synapsin I with FRAP, Huntingtin-associated protein-1 (HAP1) was found to be a new partner of synapsin I and was involved in regulating the axonal transport of synapsin I-containing vesicles [56]. Another presynaptic protein, $\mathrm{GABA}_{\mathrm{B}}$ receptor, has been reported to regulate neurotransmitter release [55]. By labeling two subunits of $\mathrm{GABA}_{\mathrm{B}}$ receptor with a FRET pair, CFP and YFP, respectively, Laviv et al. found that high FRET efficiency was associated with low SVs release probability, which suggested an important role of $\mathrm{GABA}_{B}$ receptor played in mediating SVs release [44]. Furthermore, some other proteins tagged with FPs have also been used to explore the dynamics of SVs. For example, EGFP-DOC2B was used to study the prime process [25]. The GFP-vesicular monoamine transporter and GFP-vesicular acetylcholine transporter were used to view the release of relative neurotransmitters [76]. Thanks to FRAP, a faster process of endocytosis mediated by clathrin could be seen with clathrin-EGFP/mCherry application [66].

\section{Conclusions}

It is clear that SVs are indispensable for the information transfer between neurons, and a large number of proteins participate in the regulation of this process. How do SVs travel from soma to neural terminal and fuse to the presynaptic membrane or exchange among different SVs pools? What kinds of modes do there usually exist in the cycling of SVs recovery? These questions remain unclear while partly being answered with the help of FPs in this review. There is no doubt that FPs technology has widened the research field of life sciences and advanced our understanding of the presynaptic structure and function since the first GFP was discovered. FPs provide a powerful tool for direct observation and quantification of cellular processes in living cells [11]. They are usually applied to view the distribution of proteins [33], track the migration of SVs [83], and study protein activity during stimulations in presynaptic termi- 
nal [35]. Applications of both target proteins labeled with FPs and relative fluorescent technologies allow us to follow the fate of an individual vesicle at synapses with high spatial and temporal resolution, varying from the formation, cluster, transport, dock, prime and release. In addition, FPs also contribute to exploring the roles which SVs-associated proteins play in the regulation of the movement or recycling of SVs. Such fluorescent probes are convenient for us to study the dynamics of SVs visually especially on a molecularly specific level [1]. However, the fidelity and accuracy are limited when the biosensor is applied to visualize thick tissues. In addition, the fluorescent decay, which will cause deviation, cannot be avoided once utilizing $\mathrm{pH}$-sensitive FPs. This shortcoming has to be taken into consideration if this kind of FP is employed to explore the fast process like exo- or endocytosis. Furthermore, there are still some limitations in detecting the multiple molecular signals simultaneously, which means more colorful biosensors are needed to be developed. Hopefully, with advanced imaging devices and novel or improved biosensors, the kinetics of SVs can be studied more accurately and deeply.

\section{Acknowledgements}

This work was supported in part by grants from the National Natural Science Foundation of China (NSFC) 31670867, No. 31670961.

\section{Disclosure}

The authors report no conflict of interest.

\section{References}

1. Afuwape OA, Kavalali ET. Imaging synaptic vesicle exocytosisendocytosis with ph-sensitive fluorescent proteins. Methods Mol Biol 2016; 1474: 187-200.

2. Alabi AA, Tsien RW. Synaptic vesicle pools and dynamics. Cold Spring Harb Perspect Biol 2012; 4: a013680.

3. Amaral E, Guatimosim S, Guatimosim C. Using the fluorescent styryl dye fm1-43 to visualize synaptic vesicles exocytosis and endocytosis in motor nerve terminals. Light Microscopy: Methods and Protocols 2011; 137-148.

4. Aravanis AM, Pyle JL, Tsien RW. Single synaptic vesicles fusing transiently and successively without loss of identity. Nature 2003; 423: 643-647.

5. Arthur CP, Stowell MH. Structure of synaptophysin: A hexameric marvel-domain channel protein. Structure 2007; 15: 707-714.

6. Astigarraga S, Hofmeyer K, Farajian R, Treisman JE. Three drosophila liprins interact to control synapse formation. J Neurosci 2010; 30: 15358-15368.

7. Atherton JF, Bevan MD. Ionic mechanisms underlying autonomous action potential generation in the somata and dendrites of gabaergic substantia nigra pars reticulata neurons in vitro. J Neurosci 2005; 25: 8272-8281.

8. Balaji J, Ryan TA. Single-vesicle imaging reveals that synaptic vesicle exocytosis and endocytosis are coupled by a single stochastic mode. Proc Natl Acad Sci U S A 2007; 104: 20576-20581.

9. Bancaud A, Huet S, Rabut G, Ellenberg J. Fluorescence perturbation techniques to study mobility and molecular dynamics of proteins in live cells: Frap, photoactivation, photoconversion, and flip. Cold Spring Harb Protoc 2010; 2010: pdb.top90.

10. Barondeau DP, Kassmann CJ, Tainer JA, Getzoff ED. Structural chemistry of a green fluorescent protein zn biosensor. J Am Chem Soc 2002; 124: 3522-3524.

11. Bialecka-Fornal M, Makushok T, Rafelski SM. A review of fluorescent proteins for use in yeast. Methods Mol Biol 2016; 1369: 309-346.

12. Brejc K, Sixma TK, Kitts PA, Kain SR, Tsien RY, Ormo M, Remington SJ. Structural basis for dual excitation and photoisomerization of the aequorea victoria green fluorescent protein. Proc Natl Acad Sci U S A 1997; 94: 2306-2311.

13. Cano R, Ruiz R, Shen C, Tabares L, Betz WJ. The functional landscape of a presynaptic nerve terminal. Cell Calcium 2012; 52: 321-326.

14. Cazares VA, Njus MM, Manly A, Saldate JJ, Subramani A, BenSimon Y, Sutton MA, Ashery U, Stuenkel EL. Dynamic partitioning of synaptic vesicle pools by the snare-binding protein tomosyn. J Neurosci 2016; 36: 11208-11222.

15. Chapman ER. How does synaptotagmin trigger neurotransmitter release? Annu Rev Biochem 2008; 77: 615-641.

16. Chen H, Puhl HL, 3rd, Koushik SV, Vogel SS, Ikeda SR. Measurement of fret efficiency and ratio of donor to acceptor concentration in living cells. Biophys J 2006; 91: L39-41.

17. Chen Y, Song X, Ye S, Miao L, Zhu Y, Zhang RG, Ji G. Structural insight into enhanced calcium indicator gcamp3 and gcampj to promote further improvement. Protein Cell 2013; 4: 299-309.

18. Chi P, Greengard P, Ryan TA. Synapsin dispersion and reclustering during synaptic activity. Nat Neurosci 2001; 4: 1187-1193.

19. Daste F, Galli T, Tareste D. Structure and function of longin snares. J Cell Sci 2015; 128: 4263-4272.

20. Davie JT, Kole MH, Letzkus JJ, Rancz EA, Spruston N, Stuart GJ, Hausser M. Dendritic patch-clamp recording. Nat Protoc 2006; 1: $1235-1247$.

21. Drew LJ, Wood JN. Fm1-43 is a permeant blocker of mechanosensitive ion channels in sensory neurons and inhibits behavioural responses to mechanical stimuli. Molecular Pain 2007; 3: 1

22. Edwards RH. The neurotransmitter cycle and quantal size. Neuron 2007; 55: 835-858.

23. Escobedo JO, Rusin O, Lim S, Strongin RM. Nir dyes for bioimaging applications. Curr Opin Chem Biol 2010; 14: 64-70.

24. Fernandez-Alfonso T, Ryan TA. The kinetics of synaptic vesicle pool depletion at cns synaptic terminals. Neuron 2004; 41: $943-$ 953.

25. Friedrich R, Groffen AJ, Connell E, van Weering JR, Gutman O, Henis YI, Davletov B, Ashery U. Doc2b acts as a calcium switch and enhances vesicle fusion. J Neurosci 2008; 28: 6794-6806.

26. Gaffield MA, Betz WJ. Imaging synaptic vesicle exocytosis and endocytosis with fm dyes. Nat Protoc 2006; 1: 2916-2921. 
27. Gale J, Marcotti W, Kennedy H, Kros C, Richardson G. Fm1-43 dye behaves as a permeant blocker of the hair-cell mechanotrans ducer channel. J Neurosci 2001; 21: 7013-7025.

28. Gao J, Hirata M, Mizokami A, Zhao J, Takahashi I, Takeuchi H, Hirata M. Differential role of snap-25 phosphorylation by protein kinases $\mathrm{a}$ and $\mathrm{c}$ in the regulation of snare complex formation and exocytosis in pc12 cells. Cell Signal 2016; 28: 425-437.

29. Hammond JW, Lu SM, Gelbard HA. Platelet activating factor enhances synaptic vesicle exocytosis via pkc, elevated intracellular calcium, and modulation of synapsin 1 dynamics and phosphorylation. Front Cell Neurosci 2015; 9: 505.

30. Han M, Zou W, Chang H, Yu Y, Zhang H, Li S, Cheng H, Wei G, Chen Y, Reinke V, Xu T, Kang L A systematic rnai screen reveals a novel role of a spindle assembly checkpoint protein bugz in synaptic transmission in c. Elegans. Front Mol Neurosci 2017; 10: 141.

31. Hara M, Zhou ZY, Hemmings HC, Jr. Alpha2-adrenergic receptor and isoflurane modulation of presynaptic ca 2 influx and exocytosis in hippocampal neurons. Anesthesiology 2016; 125: 535-546.

32. Harata NC, Aravanis AM, Tsien RW. Kiss-and-run and full-collapse fusion as modes of exo-endocytosis in neurosecretion. J Neurochem 2006; 97: 1546-1570.

33. Herzog E, Nadrigny F, Silm K, Biesemann C, Helling I, Bersot T, Steffens H, Schwartzmann R, Nagerl UV, El Mestikawy S, Rhee J, Kirchhoff F, Brose N. In vivo imaging of intersynaptic vesicle exchange using vglut1 venus knock-in mice. J Neurosci 2011; 31: 15544-15559.

34. Herzog E, Takamori S, Jahn R, Brose N, Wojcik SM. Synaptic and vesicular co-localization of the glutamate transporters vglut1 and vglut2 in the mouse hippocampus. J Neurochem 2006; 99: 1011-1018.

35. Houeland G, Nakhost A, Sossin WS, Castellucci VF. Pkc modulation of transmitter release by snap-25 at sensory-to-motor synapses in aplysia. J Neurophysiol 2007; 97: 134-143.

36. Huang CC, Yang DM, Lin CC, Kao LS. Involvement of rab3a in vesicle priming during exocytosis: Interaction with munc13-1 and munc18-1. Traffic 2011; 12: 1356-1370.

37. Iwabuchi S, Kakazu Y, Koh JY, Goodman KM, Harata NC. Examination of synaptic vesicle recycling using fm dyes during evoked, spontaneous, and miniature synaptic activities. J Vis Exp 2014; doi: 10.3791/50557.

38. Jahn R, Scheller RH. Snares - engines for membrane fusion. Nat Rev Mol Cell Biol 2006; 7: 631.

39. Kaeser PS, Deng L, Wang Y, Dulubova I, Liu X, Rizo J, Sudhof TC. Rim proteins tether ca2+ channels to presynaptic active zones via a direct pdz-domain interaction. Cell 2011; 144: 282-295.

40. Kavalali ET. The mechanisms and functions of spontaneous neurotransmitter release. Nat Rev Neurosci 2015; 16: 5-16.

41. Kavalali ET, Jorgensen EM. Visualizing presynaptic function. Nat Neurosci 2014; 17: 10-16.

42. Khvotchev M, Kavalali ET. Pharmacology of neurotransmitter release: Measuring exocytosis. Handb Exp Pharmacol 2008: 23-43.

43. Kolos YA, Grigoriyev IP, Korzhevskyi DE. A synaptic marker synaptophysin. Morfologiia 2015; 147: 78-82.

44. Laviv T, Riven I, Dolev I, Vertkin I, Balana B, Slesinger PA, Slutsky I. Basal gaba regulates gaba(b)r conformation and release probability at single hippocampal synapses. Neuron 2010; 67: 253-267.
45. Leitz J, Kavalali ET. Ca(2)(+) influx slows single synaptic vesicle endocytosis. J Neurosci 2011; 31: 16318-16326.

46. Leitz J, Kavalali ET. Fast retrieval and autonomous regulation of single spontaneously recycling synaptic vesicles. Elife 2014; 3: e03658.

47. Li H, Foss SM, Dobryy YL, Park CK, Hires SA, Shaner NC, Tsien RY, Osborne LC, Voglmaier SM. Concurrent imaging of synaptic vesicle recycling and calcium dynamics. Front Mol Neurosci 2011; 4: 34.

48. Li Y, Tsien RW. Phtomato, a red, genetically encoded indicator that enables multiplex interrogation of synaptic activity. Nat Neurosci 2012; 15: 1047-1053.

49. Li YF, Zhang XX, Duan SM. Research progress of synaptic vesicle recycling. Sheng Li Xue Bao 2015; 67: 545-560.

50. Li Z, Burrone J, Tyler WJ, Hartman KN, Albeanu DF, Murthy VN. Synaptic vesicle recycling studied in transgenic mice expressing synaptophluorin. Proc Natl Acad Sci U S A 2005; 102: 6131-6136.

51. Liao K-C, Chiu H-S, Fan S-Y, Tseng Y-D, Lu P-H. Percutaneous fiber-optic biosensor for immediate evaluation of chemotherapy efficacy in vivo (part i): Strategy of assay design for monitoring non-homogeneously distributed biomarkers. Sensors and Actuators B: Chemical 2016; 222: 544-550.

52. Lin MZ, Schnitzer MJ. Genetically encoded indicators of neuronal activity. Nat Neurosci 2016; 19: 1142-1153.

53. Liu B, Kim TJ, Wang Y. Live cell imaging of mechanotransduction. J R Soc Interface 2010; 7 Suppl 3: S365-375.

54. Liu KS, Siebert M, Mertel S, Knoche E, Wegener S, Wichmann C, Matkovic T, Muhammad K, Depner H, Mettke C, Buckers J, Hell SW, Muller M, Davis GW, Schmitz D, Sigrist SJ. Rim-binding protein, a central part of the active zone, is essential for neurotransmitter release. Science 2011; 334: 1565-1569.

55. Lujan R, Shigemoto R, Kulik A, Juiz JM. Localization of the gabab receptor $1 \mathrm{a} / \mathrm{b}$ subunit relative to glutamatergic synapses in the dorsal cochlear nucleus of the rat. J Comp Neurol 2004; 475: 36-46.

56. Mackenzie KD, Lumsden AL, Guo F, Duffield MD, Chataway T, Lim Y, Zhou XF, Keating DJ. Huntingtin-associated protein-1 is a synapsin i-binding protein regulating synaptic vesicle exocytosis and synapsin i trafficking. J Neurochem 2016; 138: 710-721.

57. Matz J, Gilyan A, Kolar A, McCarvill T, Krueger SR. Rapid structural alterations of the active zone lead to sustained changes in neurotransmitter release. Proc Natl Acad Sci U S A 2010; 107: 8836-8841.

58. Michel K, Muller JA, Oprisoreanu AM, Schoch S. The presynaptic active zone: A dynamic scaffold that regulates synaptic efficacy. Exp Cell Res 2015; 335: 157-164.

59. Mohrmann R, Dhara M, Bruns D. Complexins: Small but capable. Cell Mol Life Sci 2015; 72: 4221-4235.

60. Nagai T, Sawano A, Park ES, Miyawaki A. Circularly permuted green fluorescent proteins engineered to sense Ca2+. Proc Natl Acad Sci U S A 2001; 98: 3197-3202.

61. Nakai J, Ohkura M, Imoto K. A high signal-to-noise Ca(2+) probe composed of a single green fluorescent protein. Nat Biotechnol 2001; 19: 137-141.

62. Nikolaev M, Heggelund P. Functions of synapsins in corticothalamic facilitation: Important roles of synapsin I. J Physiol 2015; 593: 4499-4510.

63. Orenbuch A, Shalev L, Marra V, Sinai I, Lavy Y, Kahn J, Burden JJ, Staras K, Gitler D. Synapsin selectively controls the mobility of 
resting pool vesicles at hippocampal terminals. J Neurosci 2012; 32: 3969-3980.

64. Orenbuch A, Shulman Y, Lipstein N, Bechar A, Lavy Y, Brumer E, Vasileva M, Kahn J, Barki-Harrington L, Kuner T, Gitler D. Inhibition of exocytosis or endocytosis blocks activity-dependent redistribution of synapsin. J Neurochem 2012; 120: 248-258.

65. Paradiso K, Wu W, Wu LG. Methods for patch clamp capacitance recordings from the calyx. J Vis Exp 2007: 244; doi: 10.3791/244.

66. Pelassa I, Zhao C, Pasche M, Odermatt B, Lagnado L. Synaptic vesicles are "primed" for fast clathrin-mediated endocytosis at the ribbon synapse. Front Mol Neurosci 2014; 7: 91.

67. Peng X, Parsons TD, Balice-Gordon RJ. Determinants of synaptic strength vary across an axon arbor. J Neurophysiol 2012; 107 2430-2441.

68. Pennuto M, Dunlap D, Contestabile A, Benfenati F, Valtorta F. Fluorescence resonance energy transfer detection of synaptophysin $\mathrm{i}$ and vesicle-associated membrane protein 2 interactions during exocytosis from single live synapses. Mol Biol Cell 2002; 13: 2706-2717.

69. Rizo J, Xu J. The synaptic vesicle release machinery. Ann Rev Biophys 2015; 44: 339-367.

70. Rizzoli SO. Synaptic vesicle recycling: steps and principles. EMBO J 2014; 33: 788-822.

71. Saheki Y, De Camilli P. Synaptic vesicle endocytosis. Cold Spring Harb Perspect Biol 2012; 4: a005645.

72. Santos MS, Foss SM, Park CK, Voglmaier SM. Protein interactions of the vesicular glutamate transporter vglut1. PLoS One 2014; 9: e109824.

73. Saroussi $S$, Nelson N. The little we know on the structure and machinery of V-ATPase. J Exp Biol 2009; 212: 1604-1610.

74. Shimomura O, Johnson FH, Saiga Y. Extraction, purification and properties of aequorin, a bioluminescent protein from the luminous hydromedusan, aequorea. J Cell Comp Physiol 1962; 59: 223-239.

75. Shin $\mathrm{OH}$. Exocytosis and synaptic vesicle function. Compr Physiol 2014; 4: 149-175.

76. Shoji-Kasai Y, Itakura M, Kataoka M, Yamamori S, Takahashi M Protein kinase C-mediated translocation of secretory vesicles to plasma membrane and enhancement of neurotransmitter release from PC12 cells. Eur J Neurosci 2002; 15: 1390-1394.

77. Siksou L, Silm K, Biesemann C, Nehring RB, Wojcik SM, Triller A, El Mestikawy S, Marty S, Herzog E. A role for vesicular glutamate transporter 1 in synaptic vesicle clustering and mobility. Eur J Neurosci 2013; 37: 1631-1642.

78. Stan A, Pielarski KN, Brigadski T, Wittenmayer N, Fedorchenko O, Gohla A, Lessmann V, Dresbach T, Gottmann K. Essential cooperation of N-cadherin and neuroligin-1 in the transsynaptic control of vesicle accumulation. Proc Natl Acad Sci U S A 2010; 107: 11116-11121.

79. Sudhof TC. Neurotransmitter release: The last millisecond in the life of a synaptic vesicle. Neuron 2013; 80: 675-690.

80. Sudhof TC. The presynaptic active zone. Neuron 2012; 75: 11-25.

81. Sudhof TC. The synaptic vesicle cycle. Annu Rev Neurosci 2004; 27: 509-547.

82. Sudhof TC, Rothman JE. Membrane fusion: Grappling with snare and sm proteins. Science 2009; 323: 474-477.

83. Suyama S, Hikima T, Sakagami H, Ishizuka T, Yawo H. Synaptic vesicle dynamics in the mossy fiber-CA3 presynaptic terminals of mouse hippocampus. Neurosci Res 2007; 59: 481-490.
84. Tajika Y, Takahashi M, Khairani AF, Ueno H, Murakami T, Yorifuji $\mathrm{H}$. Vesicular transport system in myotubes: Ultrastructural study and signposting with vesicle-associated membrane proteins. Histochem Cell Biol 2014; 141: 441-454.

85. Tsien RY. Building and breeding molecules to spy on cells and tumors. FEBS Lett 2005; 579: 927-932.

86. Umeda T, Okabe S. Visualizing synapse formation and remodeling: Recent advances in real-time imaging of CNS synapses. Neurosci Res 2001; 40: 291-300.

87. Valtorta F, Pennuto M, Bonanomi D, Benfenati F. Synaptophysin: leading actor or walk-on role in synaptic vesicle exocytosis? Bioessays 2004; 26: 445-453.

88. van Stegen B, Dagar S, Gottmann K. Release activity-dependent control of vesicle endocytosis by the synaptic adhesion molecule N-cadherin. Sci Rep 2017; 7: 40865.

89. Wachter RM, Yarbrough D, Kallio K, Remington SJ. Crystallographic and energetic analysis of binding of selected anions to the yellow variants of green fluorescent protein. J Mol Biol 2000; 301: 157-171.

90. Wagner OI, Esposito A, Kohler B, Chen CW, Shen CP, Wu GH, Butkevich E, Mandalapu S, Wenzel D, Wouters FS, Klopfenstein DR. Synaptic scaffolding protein SYD-2 clusters and activates kinesin-3 UNC-104 in C. elegans. Proc Natl Acad Sci U S A 2009; 106: 19605-19610.

91. Wallrabe H, Periasamy A. Imaging protein molecules using fret and flim microscopy. Curr Opin Biotechnol 2005; 16: 19-27.

92. Wang Y, Shyy JY, Chien S. Fluorescence proteins, live-cell imaging, and mechanobiology: Seeing is believing. Annu Rev Biomed Eng 2008; 10: 1-38.

93. Wrosch JK, Groemer TW. Functional viability: measurement of synaptic vesicle pool sizes. Methods Mol Biol 2017; 1601: 195-204.

94. Wyatt RM, Balice-Gordon RJ. Heterogeneity in synaptic vesicle release at neuromuscular synapses of mice expressing synaptophluorin. J Neurosci 2008; 28: 325-335.

95. Xia Z, Zhou Q, Lin J, Liu Y. Stable snare complex prior to evoked synaptic vesicle fusion revealed by fluorescence resonance energy transfer. J Biol Chem 2001; 276: 1766-1771.

96. Xu J, Luo F, Zhang Z, Xue L, Wu XS, Chiang HC, Shin W, Wu LG. SNARE proteins synaptobrevin, SNAP-25, and syntaxin are involved in rapid and slow endocytosis at synapses. Cell Rep 2013; 3: 1414-1421.

97. Xue L, Mei YA. Synaptic vesicle recycling at the calyx of held. Acta Pharmacol Sin 2011; 32: 280-287.

98. Xue L, Sheng J, Wu XS, Wu W, Luo F, Shin W, Chiang HC, Wu LG. Most vesicles in a central nerve terminal participate in recycling. J Neurosci 2013; 33: 8820-8826.

99. Yu W, Kawasaki F, Ordway RW. Activity-dependent interactions of NSF and SNAP at living synapses. Mol Cell Neurosci 2011; 47: 19-27.

100. Zhao C, Dreosti E, Lagnado L. Homeostatic synaptic plasticity through changes in presynaptic calcium influx. J Neurosci 2011; 31: 7492-7496.

101. Zhu Y, Xu J, Heinemann SF. Two pathways of synaptic vesicle retrieval revealed by single-vesicle imaging. Neuron 2009; 61: 397-411.

102. Zoltowska KM, Maesako M, Lushnikova I, Takeda S, Keller LJ, Skibo G, Hyman BT, Berezovska O. Dynamic presenilin 1 and synaptotagmin 1 interaction modulates exocytosis and amyloid beta production. Mol Neurodegener 2017; 12: 15. 